\title{
Atomic Scale In Situ Electron Microscopy: Challenges and Opportunities
}

Joerg R. Jinschek

FEI Company, Materials Science BU, Eindhoven, The Netherlands.

Ongoing strong research focus on more efficient energy use and conversion, on more efficient transportation, and on environmental protecting technologies relies heavily on the advancement of (new) functional nanomaterials and nanosystems. At any stage in research and development, studies of these nanomaterials' structure, properties, and function are critical, including detailed atomic-scale insights.

To our advantage, atomic scale electron microscopy (EM), markedly advanced by utilizing recent hardand software improvements, has become a powerful and indispensable tool for characterizing those nanostructures. Ongoing activities concentrate on methodological aspects of state-of-the-art EM and thereby open routes towards atom sensitive imaging of nanostructures that play a crucial role in numerous applications.

However, the actual state and function of nanomaterials 'in operation' cannot always be inferred from examination under standard EM high vacuum and room temperature conditions or from postmortem EM studies. In situ techniques enable visualization of structural evolution under operational (or environmental) conditions, thereby providing new insights in important materials science questions [1].

EM imaging schemes will be discussed that focus on maintaining spatial resolution in in situ studies, while controlling electron beam / structure interactions. For instance, the inherent atomic scale microscope resolution can indeed be retained in an aberration-corrected environmental microscope (ETEM) under optimized electron illumination conditions, enabling environmental studies in e.g. oxidation, reduction, or corrosion experiments [1-4].

Further development in in situ EM stages will be highlighted as well. As one example, the NanoEx-i/v heating holder - based on MEMS technology - enables faster settling time with a more accurate knowledge of experimental conditions (Fig 2a). Fine temperature control with an advanced closed loop control using a four-probe measurement of the heater's resistance enables quantitative atomic-scale studies at elevated temperatures in any (gaseous) environment [5]. NanoEx-i/v also allows analytical EDS, utilizing the four detector SuperX EDS system [6], for compositional studies at elevated temperatures (Fig 2b). Additional fours electrical contacts allow sample biasing even during heating experiments.

Moreover, the integration of a heater into a gas-flow MEMS nanoreactor enables operando EM combining structural characterization of e.g. catalytic materials with simultaneous measurement of its activity for gaseous reactions [7]. These advancements open up for unprecedented control over experimental conditions in advanced EM experiments of dynamic phenomena in materials sciences. 


\section{References:}

[1] J. R. Jinschek, Chemical Communications 50 (2014), p. 2696.

[2] J. R. Jinschek et al., Micron 43 (2012), p. 1156.

[3] S. Helveg, et al., Micron 68 (2015), p. 176.

[4] H. Yoshida et al., Science 335 (2012), p. 317.

[5] L. Mele, et al., Microscopy Research and Technique (2016), DOI: 10.1002/jemt.22623.

[6] P. Schlossmacher et al., Microscopy Today 18 (2010), p. 14.

[7] S.B. Vendelbo, et al., Nature Materials 13 (2014), p. 884.

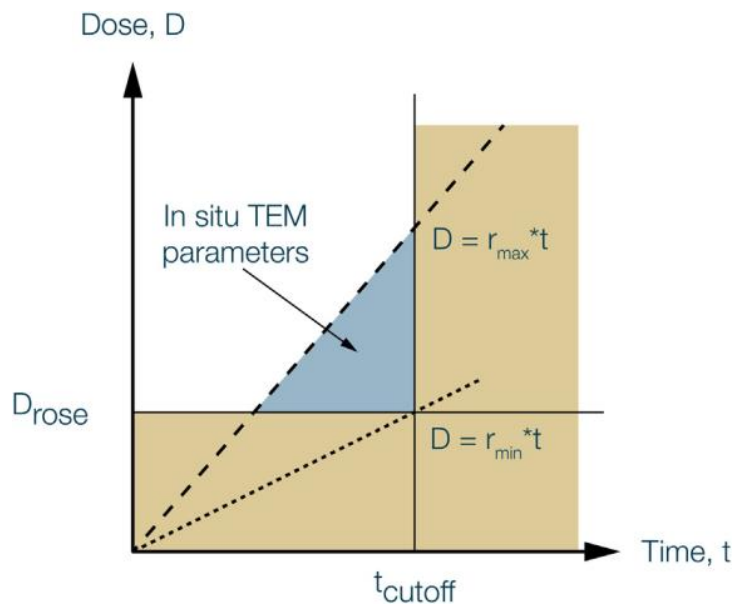

Figure 1. Schematics of the experiment parameter window of electron dose, electron dose-rate and exposure time enabling high-resolution in situ EM observations under non-invasive illumination conditions (see details in [3]).

(a)

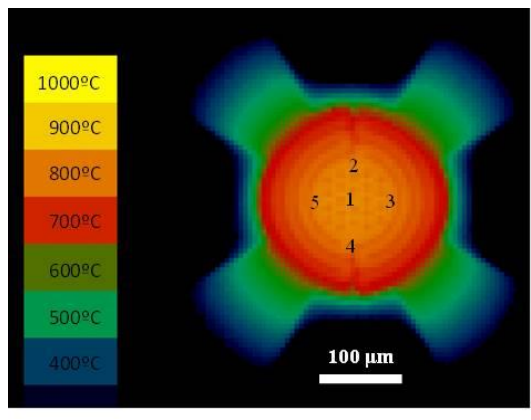

\begin{tabular}{|c|c|}
\hline Position & Absolute T (으) \\
\hline 1 & 821 \\
\hline 2 & 817 \\
\hline 3 & 819 \\
\hline 4 & 813 \\
\hline 5 & 821 \\
\hline
\end{tabular}

(b)

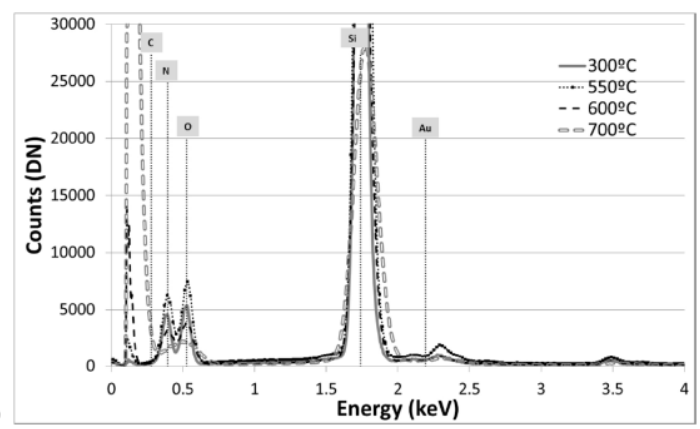

Figure 2. (a) Thermal imaging of the NanoEx-i/v MEMS microheater indicating the uniform temperature distribution over the 'sample' area; (b) EDS spectra showing peak broadening at higher temperature and the appearance of a strong background signal at $700^{\circ} \mathrm{C}$ for energy lower than $400 \mathrm{eV}$, which limits the detection of light elements in heated EDS experiments (see details in [5]). 